\title{
High power fiber MOPA based QCW laser delivering pulses with arbitrary duration on demand at high modulation bandwidth
}

\author{
Rok Petkovšek, ${ }^{1, *}$ Vid Novak, ${ }^{2}$ and Vid Agrež ${ }^{1}$ \\ ${ }^{1}$ Faculty of Mechanical Engineering, University of Ljubljana, Aškerčeva 6, SI-1000 Ljubljana, Slovenia \\ ${ }^{2}$ LPKF Laser and Elektronika d.o.o., Polica 33,4202 Naklo, Slovenia \\ rok.petkovsek@fs.uni-lj.si
}

\begin{abstract}
We report on a concept of a fiber MOPA based quasi-CW laser working at high modulation bandwidths up to $40 \mathrm{MHz}$ capable of producing arbitrary pulse durations at arbitrary repetition rates. An output power of over $100 \mathrm{~W}$ was achieved and an on-off contrast of $25 \mathrm{~dB}$. The laser features a dual-channel (dual-wavelength) seed source, a double stage YDF amplifier and a volume-Bragg-grating-based signal de-multiplexer. Minimization of transients was conducted through experiment and model analysis.

(C)2015 Optical Society of America

OCIS codes: (140.3538) Lasers, pulsed; (140.3510) Lasers, fiber; (140.3615) Lasers, ytterbium.
\end{abstract}

\section{References and links}

1. C. Bollig, P.-G. Hofmeister, M. Kunze, J. Schmidt, S. Fayed, and R. Reuter, "Efficient single-frequency pulsed all-fibre amplifier for coherent lidar," in 2013 Conference on Lasers and Electro-Optics- International Quantum Electronics Conference, (Optical Society of America, 2013), paper CJ P 31.

2. P. Elahi, S. Yılmaz, Y. B. Eldeniz, and F. Ö. Ilday, "Generation of picosecond pulses directly from a $100 \mathrm{~W}$, burst-mode, doping-managed Yb-doped fiber amplifier," Opt. Lett. 39(2), 236-239 (2014).

3. J. Petelin, B. Podobnik, and R. Petkovšek, "Burst shaping in a fiber-amplifier chain seeded by a gain-switched laser diode," Appl. Opt. 54(15), 4629-4634 (2015).

4. C. Larsen, K. P. Hansen, K. E. Mattsson, and O. Bang, "The all-fiber cladding-pumped Yb-doped gain-switched laser," Opt. Express 22(2), 1490-1499 (2014).

5. A. Starodoumov, L. R. M. Snadden, and A. Diening, "Fiber-mopa apparatus for delivering pulses on demand," United States Patent US20120320450 (December 20, 2012).

6. Q. Mao and J. W. Y. Lit, "Transient response of wavelength-switchable Erbium-doped fiber lasers with linear coupled cavities," Microw. Opt. Technol. Lett. 35(4), 330-333 (2002).

7. M. J. Munroe, "Method and apparatus for producing arbitrary pulsetrains from a harmonic fiber laser," United States Patent US7885298 (February 8, 2011).

8. A. Tuennermann, H. Zellmer, and J. P. Ruske, "Directly modulatable laser," United States Patent US 20010014107 (August 16, 2001).

9. R. Petkovšek and V. Agrež, "Single stage Yb-doped fiber laser based on gain switching with short pulse duration," Opt. Express 22(2), 1366-1371 (2014).

10. V. Agrež and R. Petkovšek, "Gain-switched Yb-doped fiber laser for microprocessing," Appl. Opt. 52(13), 3066-3072 (2013).

\section{Introduction}

Lasers are nowadays widely used in different areas of material processing. In laser transfer printing application a pattern is transferred onto the workpiece with a laser. The print information is encoded in a bitstream. To write the desired bitstream and fully exploit the speed of the scanning head the laser needs to deliver pulses on demand at modulation bandwidth in the range beyond $20 \mathrm{MHz}$. Typically the process requires powers in the range of hundreds of watts, high pulse to pulse stability and an on/off contrast in excess of $15 \mathrm{~dB}$.

Laser systems which meet this criteria are usually based on a continuous wave (CW) laser employing an external electro-optical or acusto-optical modulator [1] to cut out the desired 
bitstream. The disadvantage is the bulk of the laser head, which must be moved at high speeds over the workpiece, high power consumption and in the case of electro-optical modulator high voltages. Another approach found in commercial systems is to use high power laser diodes to directly modulate output. The downside in this case is lower beam quality which cannot be compared to laser systems with near single mode outputs.

An alternative for compact adaptive system is the fiber based Master Oscillator Power Amplifier (MOPA), which uses directly modulated seed diode to produce pulses on demand. Such arbitrary pulse operation mode causes temporal gain fluctuations. Usually the first part of the pulse experiences higher gain while for the rest of the pulse the gain is reduced. This was observed in MOPA lasers operating in burst mode [2] and was solved by pulse shaping at the input to produce an even output train of pulses [3]. Similar effect can be observed for pulses with longer durations and lower pulse powers. Transient behavior due to the gain change was described for the gain switched lasers [4] and it follows that the transient times can be over $500 \mu \mathrm{s}[5,6]$ if not addressed correctly.

The best way to produce arbitrary pulse durations on demand and mitigate transients is to achieve constant power output from a MOPA system or a quasi CW operation (QCW) by introducing an additional idler seed to the signal. The role of the idler is in reducing the gain buildup between signal pulses to such extent that gain for the signal pulses is kept constant. At the output the idler is filtered out, an example is frequency conversion [7], and only the amplified signal laser light is used for the processing. Additional benefit of using an idler in such systems is the suppression of Amplified Spontaneous Emission (ASE) between the signal pulses. To avoid frequency conversion element a dual channel seed source can be used in MOPA laser [8]. One channel presents the signal and another the idler. The channels have different wavelengths and orthogonal polarizations, which serves for idler filtering at MOPA output. In this case, the polarization filtering is at disadvantage because it is hard to maintain the polarization contrast at high power levels. Also the transient gain region was not considered.

In this work, a dual wavelength approach for seeding a dual-stage MOPA fiber laser has been used. The amplifier chain is constantly seeded, therefore suppressing the ASE lasing during the periods of inactive operation. This also enables the system to operate with a continuous pump source, preventing the slow response of the pump diode to affect the overall system dynamic performance. To understand and mitigate the transient behavior of the system when switching between the seed wavelengths, a numerical model was developed.

\section{Experimental setup}

The MOPA system was built around ytterbium doped active fiber with relatively broad emission peak from which the wavelengths for the idler and the signal are chosen. The limiting factor here is the wavelength dependent gain and reabsorption of the seed because of the three level nature of the ytterbium. In the system presented in this paper a signal wavelength of $1064 \mathrm{~nm}$ and the idler of $1060 \mathrm{~nm}$ were used, reasons being lower reabsorption at these wavelengths, available seed diodes and sufficient separation for the output filtering element. The experimental setup is shown in Fig. 1.

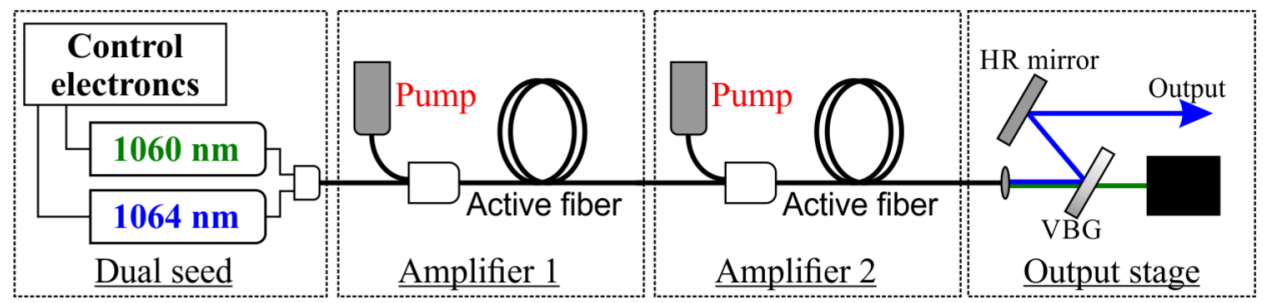

Fig. 1. Experimental setup. The VBG is Volume Bragg Grating used for filtering idler and signal. 
A pair of directly modulated laser diodes was used as the seed source for double stage ytterbium-doped amplifier (YDFA). A volume-Bragg-grating (VBG) has been used to filter the idler from the output. The seed driver consists of a field programmable gate array (FPGA)based logical driver and a power amplifier that provides the drive current for the seed laser diodes. An $18 \mathrm{~W}$ first stage pump and a $120 \mathrm{~W}$ second stage pump were used. To evaluate the amplifier response a test bitstream of alternating signal and idler pulses was launched into the amplifier chain. The signal / idler pulse modulation bandwidth was 20-40 $\mathrm{MHz}$ and minimum pulse duration of $20 \mathrm{~ns}$.

\section{Theory}

The chosen combination of the signal and the idler wavelengths are $4 \mathrm{~nm}$ apart which corresponds to emission cross section relative difference of $5 \%$. This still produces transients in the amplified output after exchanging the signal and idler when seeding with equal power. Changing the length of the amplifiers, the pump power level and the power of the seeds has a direct influence on the stable output bitstream. The intrinsic properties of the system such as effective cross section and losses also influence the output.

To gain an insight into the operation of dual wavelength seeded amplifier system a numerical model based on rate equations [9] was developed. The system of rate equations included description of upper laser level $(N)$, signal photons $\left(S_{1}\right)$ at $1064 \mathrm{~nm}$, idler photons $\left(S_{2}\right)$ at $1060 \mathrm{~nm}$, and ASE $\left(S_{3}\right)$ photons temporal variation The equations are evaluated in $n$ coupled sections [10] along the fiber to take into account the longitudinal dependence. The stimulated processes together with the schematics of the problem are presented in Fig. 2(a). Equations for $i$-th section of length $d z=L / n$ can be written:

$$
\begin{aligned}
& \dot{N}_{i}=-\frac{\Gamma c}{V_{i}} \sum_{k=1}^{3} \sigma_{k}\left(S_{k, i}^{+}+S_{k, i}^{-}\right)\left(N_{i}-N_{k}^{t r}\right)-\frac{N_{i}}{\tau_{21}}+w_{i}, \\
& \dot{S}_{k, i}^{ \pm}=\frac{\Gamma c}{V_{i}} \sigma_{k} S_{k, i}^{ \pm}\left(N_{i}-N_{k}^{t r}\right)-\frac{S_{k, i}^{ \pm}}{\tau_{c}}+\frac{S_{k, i \mp 1}^{ \pm}}{\tau_{c}}+\frac{\beta}{2} \frac{N_{i}}{\tau_{21}} .
\end{aligned}
$$
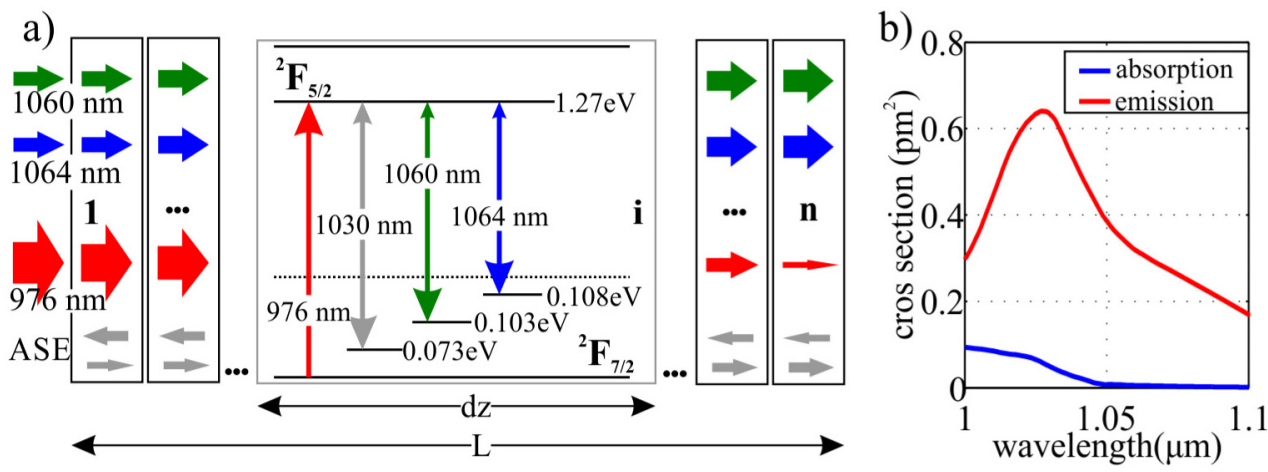

Fig. 2. Schematics (a) of a modeled problem showing stimulated processes in $i$-th segment together with low laser level energies. The calculation was done for 100 segments. The cross sections for wavelengths over $1 \mu \mathrm{m}$ are shown on (b).

The segment index $i$ runs from 1 to the number of segments $n$ and index $k$ from 1 to 3 denoting the populations of photons inside the fiber. Factor $\Gamma$ denotes the overlap integral between the laser mode and the active core of the fiber, $c$ is the speed of light in the fiber, $V_{\mathrm{i}}$ is the fiber segment volume and $\beta$ is the part of spontaneous emission coupled to the fiber. The value of $\beta$ is of the order of $1 \mathrm{e}-6$ and affects only the amplified spontaneous emission. The time of flight for a segment is denoted with $\tau_{\mathrm{c}}=d z / c$, rate of pumping by $w_{\mathrm{i}}$, upper laser 
lifetime by $\tau_{21}$, lower laser level occupancy by $N^{\mathrm{tr}}$ and stimulated emission cross section by $\sigma$. The latter two have a major effect on the stability of operation when changing the wavelength during MOPA operation in spite of the smoother region of ytterbium gain (Fig. 2(b)).

The pump rate is related to the cladding absorption in the fiber, $6 \mathrm{~dB} / \mathrm{m}$ and $7.4 \mathrm{~dB} / \mathrm{m}$ for the first and the second amplifier respectively. For optimal absorption the fiber lengths of $3 \mathrm{~m}$ and $2.6 \mathrm{~m}$ were chosen. A semi stable operation of the first amplifier stage was measured and compared with numerical model Fig. 3(a). The model and the measurement offer good agreement for pump power of $18 \mathrm{~W}$ and seed powers of $70 \mathrm{~mW}$ and $45 \mathrm{~mW}$ for signal and idler respectively. The ASE was also modeled and the result corresponds to the measured $2 \%$ of the total signal in forward direction. The additional information inferred from the model is the upper laser level population temporal variation shown in Fig. 3(b). It can be observed that the occupancy fluctuates with switching the wavelength when the bitstream is generated.
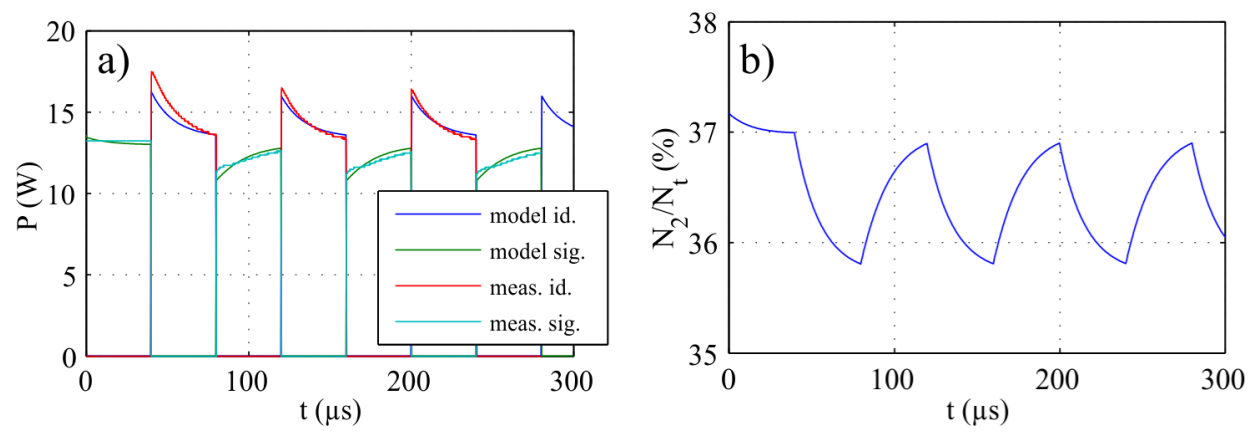

Fig. 3. Comparison (a) of the model with the measurement made for 40us pulse train of interchanging signal and idler from single amplifier. The upper laser level population is shown in (b).

Evaluating the model further shows that stable output can be achieved by controlling the input seed powers alone with minimal variation in the occupancy of the upper laser level. At the highest tested pump power of $18 \mathrm{~W}$ of the first amplifier stage the idler seed power must be $83 \%$ of the signal seed power to compensate for different stimulated emission cross sections thermal occupancy of the lower laser level and in consequence the gain.

\section{Experimental results and discussion}

A series of tests were conducted to experimentally determine the amplifier chain response to the input bitstream and to find the optimal mode of operation. The bitstream consisted of four series of alternating signal and idler pulses with durations spanning across three orders of magnitude (from $400 \mathrm{~ns}$ to $400 \mu \mathrm{s}$ ). The duration of the test bitstream was $2 \mathrm{~ms}$. It was found through experiment that all the relevant dynamic components of the amplifier response can be invoked this way. Maximum output powers of $13 \mathrm{~W}$ and $105 \mathrm{~W}$ has been achieved in the first and second stage respectively with an optical-to-optical conversion efficiency of $70 \%$ in the first stage and $82 \%$ in the second stage.

The response to the seeded bitstream was evaluated in terms of the relative stability of the signal component output power by introducing three response variables: the relative overshot $\left(\boldsymbol{R O}=P_{\text {out,max }} / P_{\text {out,ref }}-1\right)$, relative undershot $\left(\boldsymbol{R U}=P_{\text {out,min }} / P_{\text {out,ref }}-1\right)$ and the min-to-max relative difference $(\boldsymbol{M M R D}=\boldsymbol{R} \boldsymbol{O}-\boldsymbol{R} \boldsymbol{U})$. The stationary power level of the signal $(1064 \mathrm{~nm})$ component has been chosen as the reference value $P_{\text {out,ref, }}$, whereas $P_{\max }$ and $P_{\min }$ are the maximum and minimum output power in the amplifier response to the individual test bitstream.

The minimization of $\boldsymbol{M M R D}$ was chosen as the optimization criterion. With the help of numerical model three main optimization parameters were identified: the signal seed power $\left(P_{\mathrm{s} 1}\right)$, the idler seed power $\left(P_{\mathrm{s} 2}\right)$ and the first stage (YDFA1) pump power $\left(P_{\mathrm{p} 1}\right)$. The upper 
values of the $P_{\mathrm{s} 1}, P_{s 2}$ and $P_{\mathrm{p} 1}$ parameters were limited by the available power of the seed and the pump diodes and by the transmission losses in the fiber-based delivery sub-system. The lower values of the $P_{\mathrm{s} 1}$ and $P_{\mathrm{s} 2}$ parameters have been limited by the onset of ASE in the YDFA1. The lower value of the $P_{\mathrm{p} 1}$ parameter has been limited by the onset of ASE in YDFA2. The output power of the YDFA2 was not considered as an optimization parameter, since we were interested in operating the laser at various levels of output power.

Due to the vast optimization parameter space, only the $P_{\mathrm{s} 1}$ parameter was varied during the experiments, whereas the value of the other two parameters $\left(P_{\mathrm{s} 2}\right.$ and $\left.P_{\mathrm{p} 1}\right)$ was kept constant. The values of these two parameters were set to their maximum values $(45 \mathrm{~mW}$ and $18 \mathrm{~W}$ respectively). The onset of ASE in YDFA1 occurs at $P_{\mathrm{s} 1}$ values lower than $25 \mathrm{~mW}$, while the maximum available value of $P_{\mathrm{s} 1}$ was $70 \mathrm{~mW}$.

The results of the response analysis are shown in Fig. 4 as contour plots of the response variables $\boldsymbol{R} \boldsymbol{O}$ in 4(a), $\boldsymbol{R} \boldsymbol{U}$ in 4(b) and $\boldsymbol{M M R D}$ in 4(c) as a function of the optimization parameter $P_{\mathrm{s} 1}$ and the YDFA2 output power $P_{\text {out }}$. It can be seen from Fig. 4 that $\boldsymbol{R} \boldsymbol{O}$ decreases with decreasing $P_{\mathrm{s} 1}$ and increasing $P_{\text {out }}$, whereas the absolute value of $\boldsymbol{R} \boldsymbol{U}$ increases with decreasing $P_{s l}$ and increasing $P_{\text {out }}$. Decreasing the overshot therefore increases the undershot and vice versa. Global minimum value of $\boldsymbol{M M R D}$ is $11 \%$, located at approximately $\left(P_{\mathrm{s} 1}=50\right.$ $\left.\mathrm{mW}, P_{\text {out }}=73 \mathrm{~W}\right)$. At full output power, the minimum value of $\boldsymbol{M M R \boldsymbol { D }}$ is $15 \%(\boldsymbol{R O}=7 \%$, $\boldsymbol{R} \boldsymbol{U}=-8 \%$ ), located approximately at $P_{\mathrm{s} 1}=45 \mathrm{~mW}$. At the lowest output power of $34 \mathrm{~W}$, the optimum value of $P_{\mathrm{s} 1}$ is approximately $40 \mathrm{~mW}$. The optimum value of $P_{\mathrm{s} 1}$ therefore also depends on the amplifier gain. This theoretically predicted and experimentally confirmed dependence can be directly used in an open-loop compensation module of the FPGA-based seed driver.
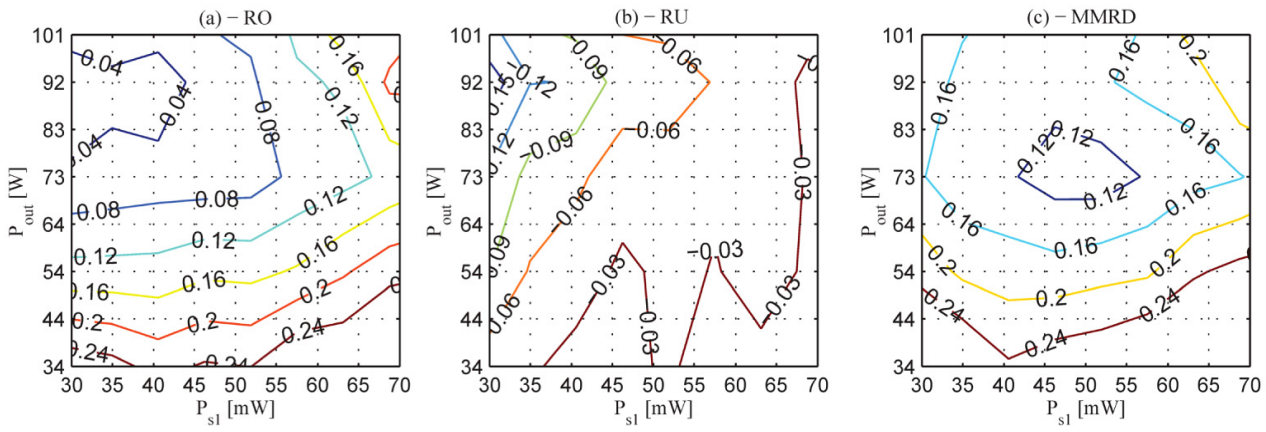

Fig. 4. A contour plot of the amplifier response. The power of the idler component and the first stage pumping power were kept constant at their maximum available levels of $P_{\mathrm{s} 2}=45 \mathrm{~mW}$ and $P_{\mathrm{p} 1}=18 \mathrm{~W}$. (a) - relative overshot in the amplified response to the test bitstream; (b) - relative undershot in the amplified response to the test bitstream; (c) - min-to-max relative difference

Although a minimization of MMRD was here used as the optimization criterion, a different criterion could be used for a specific process. In Fig. 5, a time evolution of the normalized output power is shown for $P_{\mathrm{s} 1}=45 \mathrm{~mW}, \mathrm{P}_{\text {out }}=73 \mathrm{~W}$ in Fig. $5(\mathrm{a})$ and for $P_{\mathrm{s} 1}=70$ $\mathrm{mW}, P_{\text {out }}=73 \mathrm{~W}$ in Fig. $5(\mathrm{~b})$. The same bitstream was applied by the seed driver, consisting of four sequences $(30 \times 4 \mu \mathrm{s}, 300 \times 0.4 \mu \mathrm{s}, 1 \times 400 \mu \mathrm{s}$ and $3 \times 40 \mu \mathrm{s})$ of alternating signal and idler pulses. The signal (red) and idler (blue) component are plotted on the same graph. The stationary power value of the signal component was chosen as the reference value for both components. The test bitstream duration is $2 \mathrm{~ms}$. Note that the magnitude of the relative overshot and undershot in the idler component can be ignored, due to the fact that this component is rejected in the output stage. Also, the overshot of the idler is small enough not to cause damage in the amplifier chain.

It can be seen that not only the magnitudes of $\boldsymbol{R} \boldsymbol{O}$ and $\boldsymbol{R} \boldsymbol{U}$ change with $P_{\mathrm{s} 1}$, but also the time offset where the minimum/maximum power value in the test response occurs. This time 
offsets correspond to the duration of the individual pulse and the location of the pulse in the sequence. Comparing the Figs. 5(a) and 5(b) it can be seen that changing the value of the $P_{\mathrm{s} 1}$ parameter has a different effect on the undershot and overshot for each of the four sequences of pulses. In a specific application, the effect of the overshot on the underlying process might not be the same as the effect of the undershot.

Furthermore, it might be preferential to optimize the process for a specific pulse duration. Optimization criterion, and consequently the optimal dependence of $P_{s l}$ on $P_{\text {out }}$, is therefore application specific. Using the numerical model together with the findings of the experimental work allows for quicker adaptation of the system to the specific application task.

The measurement analysis also shows that the dual wavelength seeds and input optimization effectively reduces the transient duration to under $100 \mu \mathrm{s}$.

(a)

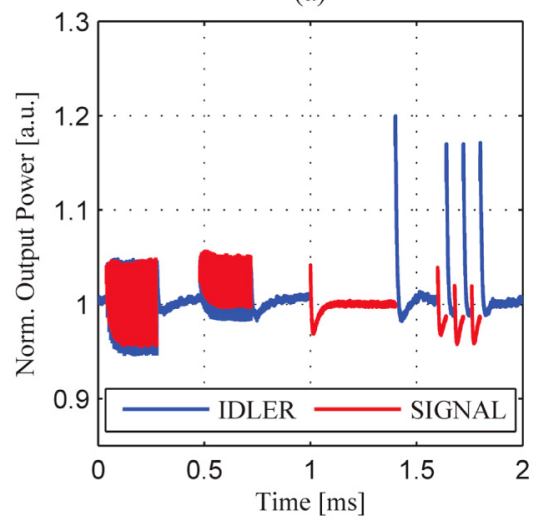

(b)

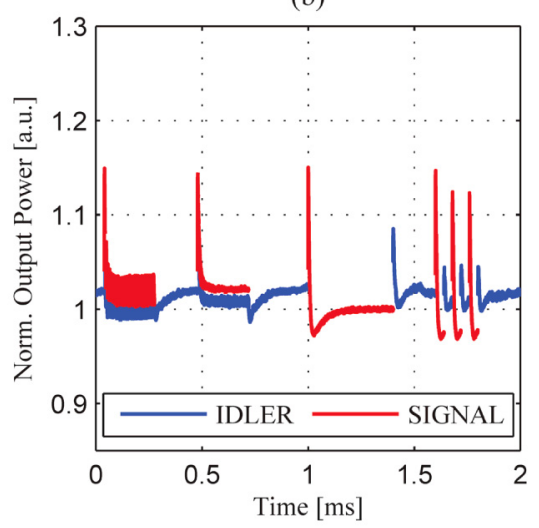

Fig. 5. Normalized output power. (a) - Amplified response at $(P \mathrm{~s} 1=45 \mathrm{~mW}, P$ out $=73 \mathrm{~W})$, where global minimum of MMRD is reached; (b) - Amplified response at $(P \mathrm{~s} 1=70 \mathrm{~mW}$, Pout $=73 \mathrm{~W})$

\section{Conclusion}

To conclude, we have demonstrated a concept of a fiber MOPA QCW laser capable of delivering pulses on demand at a modulation bandwidth of up to $40 \mathrm{MHz}$ and an output power over $100 \mathrm{~W}$. A wavelength-based multiplexer-amplifier-demultiplexer scheme has been used with a VBG-based demultiplexer that provides an on/off contrast of $25 \mathrm{~dB}$. An output beam quality of $\mathrm{M}^{2}<1.5$ was achieved for the optimized conditions, which shows a significant advantage over the high power diode lasers, but still offers a room for further improvement.

A theoretical assessment of the controllable parameters was made with the numerical model for simulating the amplifier response in a dual-wavelength operation. The effect of the operation on the inversion population was shown and the seeds input powers were identified as the main parameters for achieving a stable output.

The numerical model and its predictions were verified by comparison to the measurements done on the experimental setup. Also an overshot and undershot transient behavior were experimentally analyzed. A test bitstream was used as an input to the seed driver in order to test the dynamic response of the double stage amplifier. When operating at minimum MMRD of $11 \%$ the transient duration was less than $100 \mu$ s through the whole test bitstream. At the expense of increasing the MMRD, the duration of the transient could be decreased even further, since we found that it increases approximately linearly with the signal seed power, from $40 \mu \mathrm{s}$ at the minimum signal seed power to $200 \mu \mathrm{s}$ at the maximum signal seed power that we used. 
An optimization procedure and optimization criterion were defined and an optimal relation between the power of the individual seed components and the pumping power of the second amplification stage were discussed. The effect of varying seed power was discussed for the individual sequences of alternating signal/idler pulses.

The presented work shows, that a dual wavelength seeded amplifier chain can produce a stable output at a high modulation bandwidth with proper optimization techniques. The concept is power scalable up to several hundred watts due to fiber MOPA design and allows for scaling of the modulation bandwidth over $100 \mathrm{MHz}$.

\section{Acknowledgments}

Part of this research was supported by the Slovenian research agency ARRS (project L26780). 